\title{
Chromatin organization, meiotic status and meiotic competence acquisition in mouse oocytes from cultured ovarian follicles
}

\author{
L. D. Johnson ${ }^{1}$, D. F. Albertini ${ }^{2}$, L. K. McGinnis ${ }^{3}$ and J. D. Biggers ${ }^{3 *}$
}

${ }^{1}$ Department of Comparative Pathology, New England Regional Primate Research Center, 1 Pinehill Drive, Southborough, MA 01772, USA; ${ }^{2}$ Department of Anatomy and Cellular Biology, Tufts University School of Medicine, 136 Harrison Avenue, Boston, MA 02111, USA; and ${ }^{3}$ Department of Cell Biology. Harvard Medical School, 25 Shattuck St, Boston, MA 02115, USA

\begin{abstract}
Changes in chromatin organization, meiotic status and the development of meiotic competence in oocytes retained within mouse ovarian follicles from day 0 to day 6 in culture were examined. The effects of exposure for $24 \mathrm{~h}$ to human luteinizing hormone $(\mathrm{hLH})$ during the last day in culture was also determined. Preantral follicles from 22- to 24-day-old (prepubertal) mice develop antra and undergo significant growth from day 0 to day 4 in culture, after which the growth rates slow. The statistical significance of meiotic progression was examined using exact logistical regression analysis, which is particularly useful when the data are sparse and unbalanced. The transition from rimmed to unrimmed germinal vesicle stages was found to occur between day 2 and day 4 of follicle culture and was not influenced by exposure to hLH. Treatment with hLH caused a significant increase in the proportion of intrafollicular oocytes resuming meiosis. Assays of meiotic competence performed in vitro in oocytes retrieved from cultured follicles demonstrated that the transition from an unrimmed to a rimmed state is closely coincident with the acquisition and expression of meiotic competence. Forty-six per cent of competent oocytes from follicle cultures at day 3 progressed to metaphase II. These results indicate that the follicle culture system used in these studies supports the transformation of enclosed oocytes from a precompetent to a competent state and can maintain meiotic arrest for up to 6 days in culture. However, an increasing proportion of oocytes exhibit abnormal meiotic progression with continued follicle culture beyond 4 days.
\end{abstract}

\section{Introduction}

Culture systems have been devised for the development of mouse ovarian follicles from preantral to preovulatory stages in vitro (Qvist et al., 1990; Nayuda and Osborn, 1992; Boland et al., 1993; Merriman et al., 1993; Hartshorne et al., 1994; Spears et al., 1994). Normal growth, during 6 days in culture, was assessed by daily increase in follicle size, antrum formation and ovulation in response to hLH (Boland et al, 1993). Furthermore, ovulated oocytes exhibited germinal vesicle breakdown and were surrounded by an expanded and mucified cumulus. Lactate production and steroidogenesis are observed in response to follicle stimulating hormone (hFSH), hLH and human chorionic gonadotrophin (hCG) (Nayuda and Osborn, 1992; Boland et al., 1993; Qvist et al., 1990). Oocytes recovered after follicle culture for 12 days resumed meiosis after oocyte culture, and a small proportion cleaved to the two-cell stage after fertilization (Merriman et al., 1993). Successful fertilization and embryo transfer to a surrogate mother were achieved after follicle and oocyte culture (Spears et al., 1994).

*Correspondence.

Received 10 January 1995.
The aims of this study were to determine the meiotic status of oocytes within the follicles developing in vitro using fluorochrome staining for DNA (Mattson and Albertini, 1990; Wickramasinghe et al., 1991; Hartshorne et al., 1994) and the ability of released oocytes to undergo meiotic maturation in oocyte culture medium.

\section{Materials and Methods}

Animals

Outbred CF1 non-littermate female mice (Charles River Laboratories, Wilmington, MA), 22-24-days-old (prepubertal) were housed in a temperature and light-controlled room in accordance with principles outlined by the NIH Guide for Care and Use of Laboratory Animals.

\section{Experiments}

Three experiments were performed, each with three replicates.

In Expt 1 the daily growth of follicles was observed in culture for 6 days and the effect of hLH during the last $24 \mathrm{~h}$ of 
culture was determined. In Expt 2 the effect of hLH on the meiotic status of oocytes extracted from follicles cultured for 2, 4 or 6 days was observed. Experiment 3 determined the meiotic competence of oocytes cultured for $14 \mathrm{~h}$ after extraction from follicles grown in culture for 3 and 6 days.

\section{Follicle isolation and culture}

Ovaries were removed aseptically after cervical dislocation of the mice and placed in $35 \mathrm{~mm}$ Petri dishes containing Leibovitz L-15 medium with $2 \mathrm{mmol}_{\text {glutamine }}{ }^{-1}$ (ICN Flow Biomedicals, Inc., Costa Mesa, CA) supplemented with $3 \mathrm{mg}$ BSA $\mathrm{ml}^{-1}$ (Fraction V, Sigma, St Louis, MO). Preantral follicles, including the theca layer, were dissected from ovarian tissue using two 25 gauge needles attached to $1 \mathrm{ml}$ syringes. Follicles were measured under $\times 40$ magnification with a precalibrated ocular micrometer and Olympus CK2 inverted microscope. Follicles 115-250 $\mu \mathrm{m}$ in diameter were chosen for culture.

Follicles were cultured individually in non-tissue culturetreated 96-U-well microtitre plates (Cell Wells: Corning, Corning, NY) in $25 \mu \mathrm{l}$ droplets of Minimal Essential Medium alpha (MEM; Gibco, Grand Island, NY) under $50 \mu$ l of mineral oil (Sigma; density $0.84 \mathrm{~g} \mathrm{ml}^{-1}$ ) in $5 \% \mathrm{CO}_{2}$ in air at $37^{\circ} \mathrm{C}$ for $2,3,4$ or 6 days. Medium was supplemented with the following reagents (all obtained from Sigma): $5 \mu \mathrm{g}$ bovine insulin $\mathrm{ml}^{-\mathrm{I}}, 10 \mu \mathrm{g}$ human transferrin $\mathrm{ml}^{-1}, 2 \mathrm{mmol}$ sodium pyruvate $\mathrm{I}^{-1}$, and $2 \mathrm{mmol}$ glutamine $\mathrm{I}^{-1}, 1 \%(\mathrm{v} / \mathrm{v})$ antibioticantimycotic mixture (100 $\mathrm{U}_{\text {penicillin }} \mathrm{G} \mathrm{ml}^{-1}, 100 \mu \mathrm{g}$ streptomycin $\mathrm{ml}^{-\mathrm{I}}$ and $0.25 \mu \mathrm{g}$ amphoteracin $\mathrm{B} \mathrm{ml}{ }^{-1}$ ), 1 iu hFSH $\mathrm{ml}^{-1}$ (biological potency $=8644 \mathrm{iu} \mathrm{ml}^{-1}$; a gift from S. Raiti, National Hormone and Pituitary Program) and 5\% pooled serum (collected by cardiac puncture under ether anaesthesia) from 22-24-day-old CFI female mice. Follicles were transferred to wells of fresh medium daily. Twenty-four hours before the termination of culture in Expts $I$ and 2, half of the follicles was transferred to medium supplemented with I iu hLH ml ${ }^{-1}$ (biological potency $=5100 \mathrm{iu} \mathrm{ml}^{-1}$; a gift from S. Raiti). The remaining half was transferred to fresh culture medium without hLH. Follicle diameters including the theca layer were measured each day (Expt 1) or on the first and last days of culture (Expts 2 and 3).

\section{Oocyte culture}

Oocytes released by puncture of the follicle were either fixed immediately or transferred to oocyte maturation medium (Eppig and Schroeder, 1989) consisting of MEM supplemented with $0.23 \mathrm{mmol}$ pyruvate $\mathrm{l}^{-1}, 1 \%(\mathrm{v} / \mathrm{v})$ antibiotic-antimycotic mixture, and $5 \%(\mathrm{v} / \mathrm{v})$ fetal bovine serum (FBS; Hyclone Inc., Logan, UT). Meiotic status was evaluated after $14 \mathrm{~h}$ of culture.

\section{Fluorochrome staining}

Oocytes were fixed immediately after release from follicles or after culture in vitro. Attached cumulus cells were removed by pipetting through a pulled glass pipette. Stripped oocytes were fixed in a cytoskeletal stabilizing buffer $(0.1 \mathrm{~mol}$ Pipes $\mathrm{l}^{-1}, 5 \mathrm{mmol} \mathrm{MgCl} \cdot 6 \mathrm{H}_{2} \mathrm{O} \mathrm{l}^{-1}, 2.5 \mathrm{mmol} \mathrm{EGTA} \mathrm{l}{ }^{-1}, 0.01 \%$ (v/v) aprotinin, $1 \mathrm{mmol}$ dithiothreitol $\mathrm{l}^{-1}, 50 \%(\mathrm{v} / \mathrm{v})$ deuterium oxide, $1 \mu \mathrm{mol}$ taxol $1^{-1}$ (gift of Developmental Therapeutics Program, National Cancer Institute, Bethesda, MD), 0.1\% (v/v) Triton $\mathrm{X}-100)$, and $2 \%(\mathrm{v} / \mathrm{v})$ formalin for $20 \mathrm{~min}$ at $37^{\circ} \mathrm{C}$. Fixed oocytes were stored at $4^{\circ} \mathrm{C}$ in PBS azide until stained for $10 \mathrm{~min}$ in Hoechst $33258\left(1 \mu \mathrm{g} \mathrm{ml}^{-1}\right)$ to label chromatin. Stained oocytes were mounted in $50 \%$ (v/v) PBS-sodium azide: $50 \%(\mathrm{v} / \mathrm{v})$ glycerol and gently compressed between a coverslip and microscope slide. Coverslips were sealed with clear nail polish and slides were stored at $4{ }^{\circ} \mathrm{C}$ until analysis. The meiotic status of oocytes retained within follicles was evaluated according to the degree of heterochromatin association with the nucleolus using the classification scheme of Mattson and Albertini (1990). Germinal vesicle (GV) stages were unrimmed nucleoli (stages I and II) or rimmed nucleoli (stages III and IV). Oocyte meiotic status was classified as (a) germinal vesicle breakdown including diakinesis and metaphase of meiosis I (MI), and (b) metaphase of meiosis II (MII). Abnormal meiosis included oocytes exhibiting condensed chromatin, dispersed chromosomes and fragmented oocytes.

\section{Statistical methods}

Follicle growth. The distributions of the follicle diameters for each day in each group were summarized by order statistics (median; 10, 25, 75 and 90 percentiles). These were plotted as regular box plots (Chambers et al., 1983). The observations made on successive days on each individual follicle were serially correlated, and these correlations must be allowed for in any comparison of the mean growth curves of different groups. Wishart (1938) and Rao (1965) showed that data on growth curves can be analysed by replacing the data on each individual with a regression line expressed as a linear combination of orthogonal polynomials and comparing the distributions of the separate regression coefficients. This principle was used in this study, following the methods described by Draper and Smith (1981), examining the distributions of regression coefficients using notched box plots to test whether the successive coefficients are significantly different from zero. An illustration of this procedure for the analysis of correlated time-dependent data was described by Biggers and Papaioannou (1991).

Meiotic status. In a typical experiment, each oocyte is classified into one of a series of meiotic states: germinal vesicle with unrimmed nucleolus $\left(G V_{u}\right)$, germinal vesicle with partially or completely rimmed nucleolus $\left(\mathrm{GV}_{\mathrm{r}}\right)$, metaphase I (MI), metaphase II (MII) and other (all abnormal nuclear responses) (Mattson and Albertini, 1990). Such data are categorical, arranged in a partially ordinal series (McCullagh and Nelder, 1989; Clogg and Shihadeh, 1994). It is useful to represent the responses by the nested classification shown in Table 1 . This representation provides the basis of a three-step logistical regression analysis determined by the three branch points. The first branch point divides the data into responders and nonresponders. These responses are on a nominal scale since the two categories are interchangeable. The response variable of interest is the ratio of the responders to the number in the original sample. Since no abnormal responses were observed until after the $G V_{r}$ stage, this ratio is the proportion of $G V_{u}$ 
Table 1. Classification of the meiotic responses used in the statistical analyses

\begin{tabular}{|c|c|c|c|c|}
\hline $\begin{array}{l}\text { Analysis } \\
\text { step }\end{array}$ & Data type & & Categorie & \\
\hline & \multicolumn{4}{|c|}{ Total oocytes observed $\left(G V_{t}\right)$} \\
\hline 1 & Nominal & Non-responders $\left(\mathrm{GV}_{\mathrm{u}}\right)$ & Respor & \\
\hline 2 & Nominal & & Abnormal & Normal $\left(\geq G V_{r n}\right)$ \\
\hline 3 & Ordinal & & & MI \\
\hline
\end{tabular}

MI: metaphase I; MII: metaphase II.

oocytes that progress to at least the $\mathrm{GV}_{\mathrm{r}}$ stage. The second branch point divides the group that progresses to at least the $\mathrm{GV}_{\mathrm{r}}$ stage into those that respond normally and abnormally. These responses are on a nominal scale because the two categories are also interchangeable. The response variable of interest is the ratio of the proportion of normal responders to the total number of responders. The third branch point divides the normal responders into three categories, $\mathrm{GV}_{\mathrm{r}^{\prime}}, \mathrm{MI}$ and MII. These responses are on an ordinal scale, since they represent a temporal sequence of meiotic states that are not interchangeable. Several models can be used to analyse ordinal data. The Case $\mathrm{D}$ (logits for the cumulative distribution) model of Clogg and Shihadeh (1994) was used for this part of the analysis. The cumulative responses of interest are the numbers of oocytes that reached at least $\mathrm{MI}(\mathrm{MI}+\mathrm{MII})$, and those that reached MII. The total is the sum of those that reached at least the normal $\mathrm{GV}_{\mathrm{r}}$ stage $\left(\mathrm{GV}_{\mathrm{r}}+\mathrm{MI}+\mathrm{MII}\right)$.

The results were analysed using logistic regression analysis in which the proportion of oocytes responding at each step is assumed to follow the binomial distribution (Brown and Rothery, 1993). In this type of analysis the observed proportions $(p)$ are transformed into logits, defined by:

$$
y=\operatorname{logit} p=\ln (p /(1-p))
$$

At each step a multiple regression has been fitted to the data using the log odds form of the logistic model, given in general by:

$$
\mathbf{y}=\ln (\boldsymbol{p} /(1-\boldsymbol{p}))=\mathbf{b}^{\mathrm{T}} \mathbf{x}
$$

where $\mathbf{y}$ is a vector of the log odds or logit of the proportions of oocytes progressing from $G V_{u}$ to $G V_{r}$, where $\mathbf{x}$ is a column vector of independent variables determined by the model to be fitted to the data, and $\mathbf{b}$ is a column vector of regression coefficients.

Specifically two forms of Eqn (2) were used:

$$
\begin{aligned}
& \text { Model 1: } y=b_{0}+b_{1} x_{1}+b_{2} x_{2}+b_{1,2} x_{1} x_{2} \\
& \text { Model 2: } y=b_{0}+b_{1} x_{1}+b_{2} x^{2}{ }_{1}+b_{3} x_{2}
\end{aligned}
$$

where $x_{1}$ is the time of follicle culture, and $x_{2}$ is the presence or absence of $\mathrm{hLH}$, coded 0 and 1 , respectively (Expt 2), and 0 or $14 \mathrm{~h}$ oocyte culture coded 0 and 1 , respectively (Expt 3 ), $b_{0}$ is the intercept, and $b_{i}(i \neq 0)$ is the regression coefficient. The regression coefficients estimate the magnitude of the trends in the data. Statistical tests of the significance of the regression coefficients provide estimates of the probabilities that the effects of the independent variables and their interactions are significantly different from zero. In some analyses, the median transition time (MTT) was estimated. The fiducial limits of error $(P=0.05)$ of the MTT were obtained from estimates of the variance and covariance of $b_{0}$ and $b_{1}$ in model (1) using Fieller's formula (Fieller, 1944).

Models ( 1 ) and (2) were fitted to the data using the computer package LOGXACT-TURBO, Cytel Software Corporation (Cambridge, MA), using either the method of maximum likelihood or conditional exact inference (Hirji, 1992). A full account of these procedures is given in the LogXact computer manual. The method of maximum likelihood provides estimates of the $b_{i}$ and its variance-covariance matrix. The goodness of fit of the model was tested by the deviance and by the Hosmer-Lemeshow tests of significance (Hosmer and Lemeshow (1989). Conditional exact inference gives estimates of $b_{i}$ and their $95 \%$ confidence limits. This method is particularly useful when the data matrices are sparse, since under these conditions the estimation of $\mathbf{b}$, and its variancecovariance matrix, often fails.

The estimated regression curves are shown in Figs 3-6 after transforming the fitted equations from the logit response scale to the proportions response scale, using the inverse of (I):

$$
p=e^{y} /\left(1+e^{y}\right)
$$

\section{Results}

\section{Growth characteristics of follicles in culture}

In Expt 1, the daily growth of mouse ovarian follicles cultured for 6 days was observed. Follicles were isolated from 22-24-day-old mice. Fifty per cent were exposed to hLH on day 5 and the remainder were not. The diameters of all follicles were measured initially and then daily.

The overall variation of follicle diameters initially and on each day of culture for 5 days is shown in Fig. 1, using regular 


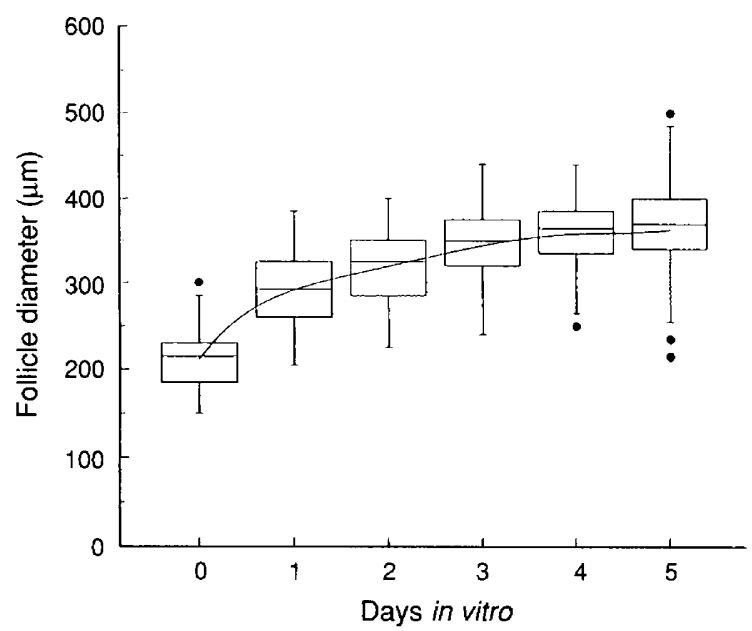

Fig. 1. Regular box plots showing the overall variation of follicle diameters initially and on each day of culture. The boxes represent the 25th, 50th (median) and 75th percentiles; the bars represent the 10th and 90 th percentiles, and the dots the outliers.

box plots. The largest increase in diameter occurred between day 0 and day 2 of culture, and little further growth was seen between day 4 and day 5. Most follicles grew between 100 and $250 \mu \mathrm{m}$, and there was a tendency for greater incremental growth to occur in smaller follicles. The estimated growth curves in the two subsequent experiments were comparable (data not shown).

The presence of an antrum and the extrusion of the oocyte were monitored on day 6 . Of the follicles in culture for 6 days, 76 of $89(85.4 \%)$ grew to $\geq 300 \mu \mathrm{m}$ (the size at which antrum formation was observed), and 29 of $89(44.1 \%)$ grew to $>400 \mu \mathrm{m}$ (the size of preovulatory follicles (Boland et al., 1993)). Extrusion of the ovum on day 6 of culture was infrequent $(6.7 \%)$.

\section{Meiotic status of oocytes from cultured follicles}

Effect of $h L H$. In Expt 2, follicles were isolated and divided into seven groups, which were allotted to one of seven treatments. The treatments formed a $2 \times 3$ factorial design with an additional control group. One factor was culture for 2,4 or 6 days; the other factor was the addition or not of LH during the last $24 \mathrm{~h}$ of culture. The additional group was uncultured follicles. The diameter of each follicle was measured at the beginning and the end of the culture period and the meiotic status of the extracted oocyte was assessed.

The meiotic status of the oocytes at the end of the experiment are summarized in Table 2 . The majority $(94.5 \%)$ of oocytes retrieved from freshly isolated follicles had unrimmed nucleoli in their germinal vesicles. After 2 days in culture, with or without exposure to hLH for the final $24 \mathrm{~h}$, only $18 \%$ of oocytes had responded normally beyond the $\mathrm{GV}_{u}$ stage. By day 4 of culture, the percentage of oocytes that had progressed from the $G V_{u}$ stage substantially increased in both groups $(60-61 \%)$. A smaller increase in the percentage of responders occurred in both groups by day $6(63-66 \%$ ). The results (Table
2) indicate that $21-37 \%$ of the total population of oocytes behave abnormally. A logistical regression analysis was done on the proportion of oocytes progressing from the $\mathrm{GV}_{\mathrm{u}}$ stage to at least the $G_{\mathrm{r}}$ stage (Step 1, Model 1). There is a highly significant effect of time of culture $\left(P<10^{-4}\right)$, no significant effect of hLH during the final $24 \mathrm{~h}$ of culture $(P=0.08)$, and no significant interaction of $\mathrm{hLH}$ concentration with time $(P=0.21)$. Thus, there is no evidence that hLH influences the rate of transition from the $\mathrm{GV}_{\mathrm{u}}$ to the $\mathrm{GV}_{\mathrm{r}}$ stage of meiosis during the last $24 \mathrm{~h}$ of culture. Figure 2 shows the cumulative distribution of the transition times from the $\mathrm{GV}_{\mathrm{u}}$ to the $\mathrm{GV}_{\mathrm{r}}$ stage of meiosis, obtained ignoring the effect of $\mathrm{hLH}$. The median transition time was found to be 2.20 [fiducial limits $(P=0.05) 1.72-2.55]$ days. The results in Table 2 also indicate that only a proportion of the oocytes that progress past the $\mathrm{GV}_{\mathrm{r}}$ stage behave normally. Further analysis of the proportion of $\mathrm{GV}_{\mathrm{r}}$ oocytes that progress normally (Step 2, Model 1) indicates that this proportion is not significantly affected by the time in culture and exposure to hLH. The proportion is approximately constant throughout the experiment and is estimated to be 0.57 [confidence limits $(P=0.05) 0.45-0.68$ ].

A small percentage of oocytes in follicles not exposed to $\mathrm{hLH}$ for the final $24 \mathrm{~h}$ of culture progressed to the MI and MII stages of meiosis (Table 2). The overall proportion of oocytes that progressed to at least $\mathrm{Ml}$ was 0.058 [confidence limits $(P=0.05) \quad 0.015-0.197]$. In contrast, there was a significant increase in the percentage of oocytes in follicles exposed to $\mathrm{hLH}$ for the final $24 \mathrm{~h}$ of culture that progressed to MI and MII stages. Analysis was done on the proportion of normally responding oocytes progressing to at least the MI stage (Step 3 , Model 1). The analysis shows a highly significant effect of hLH $\left(P=10^{-4}\right)$, no significant effect of days in culture $(P=1.0)$, and an interaction between hLH concentration and time (days) which fails to reach significance $(P=0.086)$. The calculated regression lines on days in culture are shown in Fig. 3. Regression over time in culture in the absence of hLH is not significant $(P=1.00)$. In contrast, after exposure to hLH for $24 \mathrm{~h}$, the regression of the proportion of oocytes reaching at least the MI stage was highly significantly negative $(P=0.002)$. After 2 days in culture, $87.5 \%$ reached the MI stage. However, by day 6 only $22.2 \%$ reached the MI stage.

A similar analysis of the proportion of normally responding oocytes that progressed to the MII stage (Step 3, Model 1) also showed a highly significant effect of hLH $\left(P=10^{-4}\right)$. There was a significant negative regression of days in culture in the group exposed to $h \mathrm{LH}(P=0.009$; Fig. 3). The regressions of the proportions that reach at least $\mathrm{MI}$ and those that reach MII are similar and, therefore, can be assumed to be parallel. This indicates that the proportion of oocytes that progress from MI to MII stages of meiosis is independent of days in culture.

These results show that the increase in progression from the unrimmed to rimmed nucleolar states occurred between day 2 and day 4 of culture and that the addition of hLH induced meiotic resumption. However, the response to hLH decreased over time in culture. Most follicles cultured without hLH supported transformation of oocytes to a rimmed state and maintained meiotic arrest. Therefore, the next experiment was designed to test the meiotic competence of oocytes cultured without hLH. 
Table 2. The meiotic status of oocytes in follicles from 22-24-day-old mice cultured for 0, 2, 4 and 6 days with and without stimulation on the last day of culture with hLH (1 iu $\mathrm{ml}^{-1}$ )

\begin{tabular}{|c|c|c|c|c|c|c|c|}
\hline \multicolumn{3}{|c|}{ Time of culture } & \multicolumn{5}{|c|}{ Meiotic status } \\
\hline Culture time (days) & hLH & $n$ & $\mathrm{GV}_{\mathrm{u}}$ & $\mathrm{GV}_{\mathrm{r}}$ & MI & MII & Abnormal \\
\hline 0 (control) & $0^{*}$ & 55 & $52(94.5 \%)$ & $2 \quad(3.6 \%)$ & 0 & 0 & $1 \quad(1.8 \%)$ \\
\hline 2 & 0 & 50 & $26(52.0 \%)$ & $8(16.0 \%)$ & 0 & $1 \quad(2.0 \%)$ & $15(30.0 \%)$ \\
\hline 4 & 0 & 47 & $9(19.1 \%)$ & $27(57.4 \%)$ & $1(2.1 \%)$ & 0 & $10(21.3 \%)$ \\
\hline 4 & 1 & 51 & $4(7.8 \%)$ & $17(33.3 \%)$ & $5(9.8 \%)$ & $9(17.6 \%)$ & $16(31.4 \%)$ \\
\hline 6 & 1 & 43 & 0 & $21(48.8 \%)$ & $4(9.3 \%)$ & $2 \quad(4.7 \%)$ & $16(37.2 \%)$ \\
\hline
\end{tabular}

$*_{0}=$ no hLH; $1=1 \mathrm{iu} \mathrm{hLH} \mathrm{ml}{ }^{-1}$. $n$ : number of oocytes; $\mathrm{GV}_{\mathrm{u}}$ : germinal vesicle with unrimmed nucleolus; $\mathrm{GV}_{\mathrm{r}}$ : germinal vesicle with partially or completely rimmed nucleolus; MI: metaphase I; MII: metaphase II.

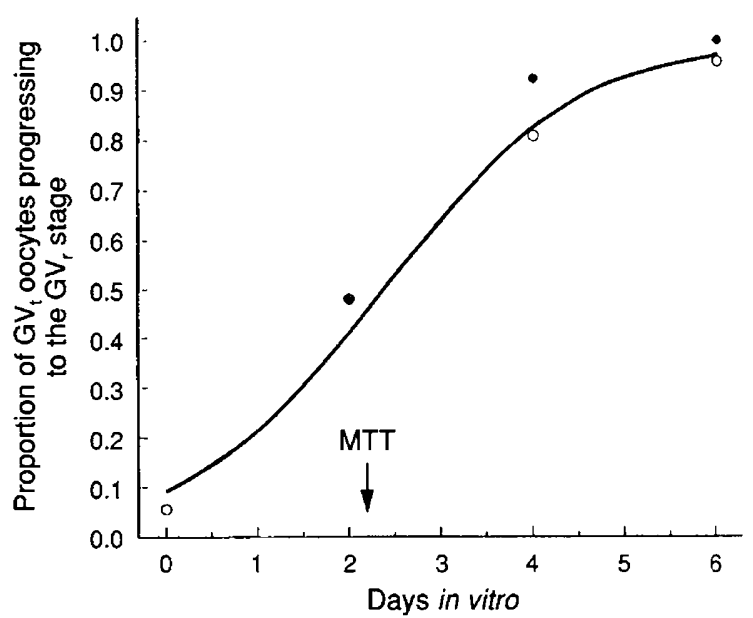

Fig. 2. The cumulative distribution of transition times of passage from the unrimmed germinal vesicle $\left(G V_{t}\right)$ stage to the rimmed $\left(G V_{\mathrm{r}}\right)$ stage of meiosis, showing the median transition time (MTT), in cultures of mouse follicles. ( ): no hLH; (๑): hLH added during the last $24 \mathrm{~h}$.

Effect of final oocyte culture: a test of meiotic competence. Isolated follicles were divided into three groups and cultured for 0,3 and 6 days. At the end of the culture period, each group was divided into two subgroups. The oocytes from one subgroup were fixed immediately to determine their meiotic status. The oocytes from the other group were cultured in oocyte maturation medium for $14 \mathrm{~h}$ and then fixed.

The meiotic status of the oocytes at the end of the experiment are summarized in Table 3 . The majority $(92.0 \%)$ of oocytes retrieved from freshly isolated follicles had unrimmed nucleoli in their germinal vesicles. Only $4 \%$ had progressed to the MI stage of meiosis. After 3 and 6 days in follicle culture, over $70 \%$ of the oocytes that were not subsequently cultured remained at the GV stage, of which $21 \%$ on day 3 and $62 \%$ on day 6 were rimmed, confirming the results of the previous experiment (Table 2). After $14 \mathrm{~h}$ in oocyte culture, most oocytes $(65 \%)$ from freshly isolated follicles remained in the GV unrimmed stage, indicating that they were meiotically incompetent. The majority of oocytes extracted from follicles after 3 and 6 days in culture resumed meiosis. The calculated

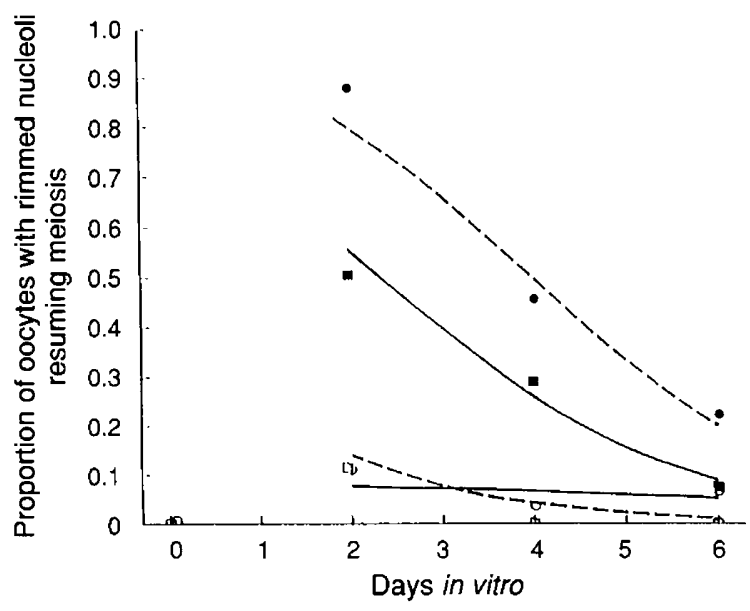

Fig. 3. The effect of hLH on the resumption and completion of meiosis. A comparison of the proportion of oocytes with rimmed nucleoli that resumed meiosis after 2, 4, or 6 days in culture with and without the addition of $\mathrm{hLH}$ for the last $24 \mathrm{~h}$ of culture. $(---)$ oocytes that progressed to at least $\mathrm{MI},(\bullet)$ : with $\mathrm{hLH},(\mathrm{)})$ : without $\mathrm{hLH} ;(-)$ oocytes that progressed to MII, (-): with hLH, (1): without hLH.

curves obtained from a logistical regression analysis (Step 1 , Model 1) are shown in Fig. 4. The median transition time is about one day longer than in the first experiment, and is estimated to be 3.32 [fiducial limits $(P=0.05) 2.64-4.24$ ] days. Subsequent oocyte culture for $14 \mathrm{~h}$ after removal from the follicle had a very marked effect on oocyte progression beyond the $G_{u}$ stage (Fig. 4). Thirty-five per cent of the oocytes isolated from freshly dissected follicles progressed to at least the $\mathrm{GV}_{\mathrm{r}}$ stage after being placed immediately in culture. However, all oocytes isolated from follicles cultured for 3 and 6 days progressed to at least the $\mathrm{GV}_{\mathrm{r}}$ stage. Analysis (Step I, Model 1) shows that this final period of oocyte culture very significantly enhanced progression to at least the $\mathrm{GV}_{\mathrm{r}}$ stage of meiosis $\left(P=10^{-4}\right)$.

The regression curve of the proportion of normal responders $\left(\mathrm{GV}_{\mathrm{r}} \mathrm{MI}, \mathrm{MII}\right)$ that progressed to at least the MI stage (Step 3, Model 1) are shown in Fig. 5. In the group whose oocytes were not cultured in isolation for a final $14 \mathrm{~h}$, a declining proportion progressed to at least the MI stage of meiosis from day 3 ( 3 of 
Table 3. The meiotic status of oocytes in follicles from 22-24-day-old mice cultured for 0,3 and 6 days with or without further culture of the isolated oocytes for $14 \mathrm{~h}$

\begin{tabular}{|c|c|c|c|c|c|c|c|}
\hline \multicolumn{3}{|c|}{ Time of culture } & \multicolumn{5}{|c|}{ Meiotic status } \\
\hline 0 & 0 & 25 & $23(92.0 \%)$ & $1 \quad(4.0 \%)$ & $1 \quad(4.0 \%)$ & 0 & 0 \\
\hline 3 & 0 & 34 & $20(58.8 \%)$ & $7(20.6 \%)$ & $1 \quad(2.9 \%)$ & $2 \quad(5.9 \%)$ & $4(11.8 \%)$ \\
\hline 6 & 0 & 34 & $3 \quad(8.8 \%)$ & $21(61.8 \%)$ & 0 & $1 \quad(2.9 \%)$ & $9(26.5 \%)$ \\
\hline 6 & 14 & 35 & 0 & $1 \quad(2.9 \%)$ & $13(37.1 \%)$ & $2(5.7 \%)$ & $19(54.3 \%)$ \\
\hline
\end{tabular}

n: number of oocytes; $\mathrm{GV}_{\mathrm{u}}$ : germinal vesicle with unrimmed nucleolus; $\mathrm{GV}_{\mathrm{r}}$ : germinal vesicle with partially or completely rimmed nucleolus; $\mathrm{MI}$ : metaphase $\mathrm{l}$; MII: metaphase II.

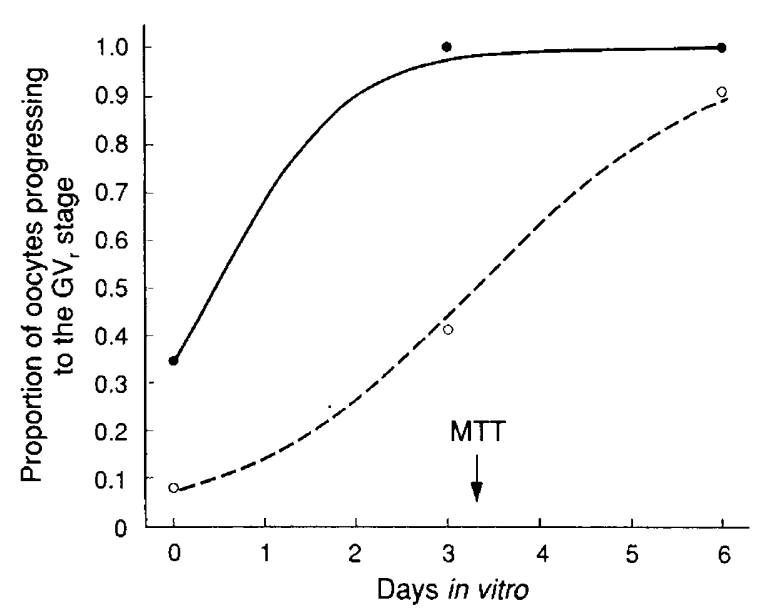

Fig. 4. A comparison of the proportion of oocytes that progressed to at least the rimmed germinal vesicle $\left(G V_{r}\right)$ stage after no follicle culture and culture for 3 or 6 days. (॰) After a further $14 \mathrm{~h}$ of oocyte culture; ( ) without oocyte culture. MTT is the estimated mean transit time from the unrimmed to the rimmed stage.

$10,30 \%$ ) to day 6 ( 1 of $22,4.5 \%$ ). The negative regression on days of follicle culture only just reaches significance $(P=0.04)$. In contrast, there was a marked increase in the proportion that progressed to at least the MI stage in the group whose oocytes were cultured for $14 \mathrm{~h}$ after isolation from their follicles $(P=0.014)$. In oocytes cultured after 3 days of follicle culture, 25 of $27(92.6 \%)$ of the oocytes had progressed to at least the MI stage of meiosis, a proportion that was maintained for 6 days ( 15 of $16,93.8 \%$ ).

The maximum progression to the MII stage occurred when the oocytes were removed from follicles cultured for 3 days $(2 / 10,20 \%)$. However, only 1 of $2(4.5 \%)$ of oocytes that were removed from follicles cultured for 6 days progressed from the MI to the MII stage. Thus, the results suggest that the proportion of oocytes progressing to MII within the cultured follicles follows a quadratic time course. The results of an analysis (Step 3, Model 2) are shown in Fig. 5. The regression on time in culture was not significant $(P=0.55)$, while the regression on the square of the time in culture was highly significant $(P=0.003)$, confirming the significance of the quadratic time course. The regression on the effect of oocyte culture is just

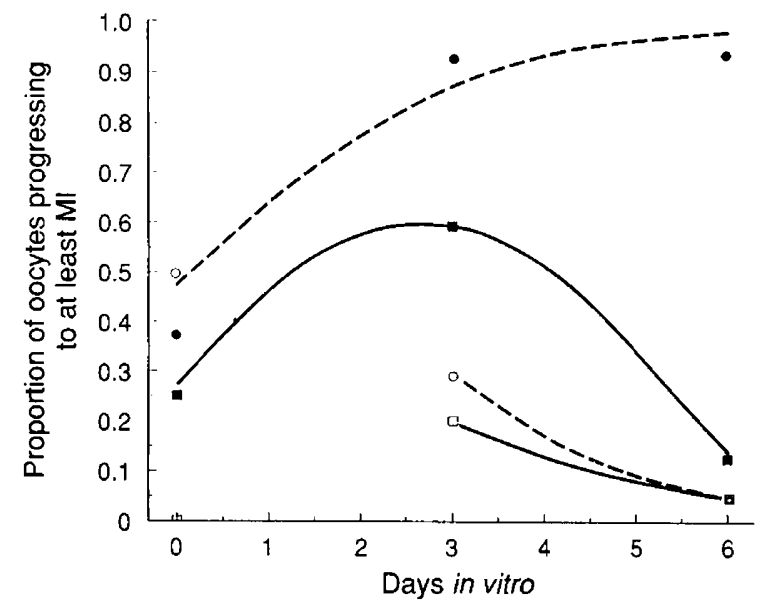

Fig. 5. The effect of oocyte culture on the resumption and completion of meiosis. (- - ) Oocytes that progressed to at least metaphase I (MI), (•) 14 h oocyte culture; ( ) no oocyte culture. (-) Oocytes that progressed to metaphase II (MII), (•) 14 h oocyte culture; ( ) no oocyte culture.

significant $(P=0.023)$. Thus, there is a significantly higher proportion of oocytes that progress from the $\mathrm{GV}_{\mathrm{r}}$ stage to the MII stage after all times of follicle culture. The results also show that there is a significantly greater proportion of oocytes progressing to the MII stage after three days of follicle culture ( 16 of $27,59.3 \%$ ) and that this proportion is significantly less after culture of follicles for 6 days ( 2 of $16,12.5 \%$ ).

Finally, an increasing proportion of abnormal meiotic forms was observed in oocytes cultured from freshly isolated follicles $(3.8 \%)$, after 3 days $(22.9 \%)$, and after 6 days $(54.3 \%)$ of follicle culture. Logistic regression analysis (Step 2, Model 1) shows that, among responders, the proportion of abnormal forms is highly significant $(P<0.0027)$. In contrast, the proportion of abnormal forms in oocytes extracted from follicles but not cultured for a further $24 \mathrm{~h}$ did not increase significantly $(P=0.81)$.

\section{Discussion}

The results of these experiments show that (i) cultured follicles support the transition of oocytes from an unrimmed to a 
rimmed GV stage between day 2 and day 4 of culture and that this transition coincides with the functional expression of meiotic competence; (ii) cultured follicles can maintain meiotic arrest of the majority of enclosed oocytes for up to 6 days in the absence of $\mathrm{hLH}$; (iii) oocyte meiotic resumption is induced in the presence of $\mathrm{hLH}$; and (iv) the fraction of oocytes capable of completing meiotic maturation is decreased after 4 days in culture. Thus, while many studies of this culture system have shown that several aspects of follicular growth and differentiation are maintained over 6 days of follicle culture, the results of the study reported here suggest that oocyte quality may diminish after 4 days.

It has been shown that nucleolar rimming occurs at the time of follicular antrum formation in vivo (Mattson and Albertini, 1990), and that this change in nuclear structure coincides with the developmental expression of meiotic competence (Wickramasinghe et al., 1991). Hartshorne et al. (1994) reported that in cultured mouse ovarian follicles, the majority of oocytes $(80 \%)$ were maintained as GV stages for up to 5 days in vitro, and that follicle growth was associated with a progressive change in chromatin organization in the oocyte from an unrimmed to a rimmed state. However, the precise timing of this transition during follicle culture was not evaluated nor was the relationship between nucleolar rimming and the expression of meiotic competence. The present studies establish that nucleolar rimming occurs between day 2 and day 4 of mouse follicle culture, when most follicular growth has been completed. Moreover, there is direct evidence that meiotic competence can be acquired over the course of follicular culture. The majority of oocytes removed from follicles cultured for at least 3 days resumed meiosis when released from the foilicles and cultured for an additional $14 \mathrm{~h}$. Since, in the absence of exogenous $\mathrm{hLH}$, most oocytes retained within follicles remain in meiotic arrest, mouse ovarian follicles cultured from the pre-antral to the antral stage must support the functional acquisition of meiotic competence and check meiotic progression, thus mimicking the two essential features of folliculogenesis in vivo.

Although studies have shown that follicular growth and function persist for up to 6 days of culture (Nayuda and Osborn, 1992; Boland et al., 1993, 1994a, b; Boland and Gosden, 1994), the question of how long oocyte function can be maintained in vitro has not been addressed in detail. In the study reported here using prepubertal animals, the transition of oocytes from a pre-competent to a competent state occurred between day 2 and day 4 , based upon nucleolar rimming and oocyte culture experiments. While $46 \%$ of oocytes cultured after culture of follicles for 3 days progressed to MII, oocytes from the follicles cultured for 6 days were largely arrested at MI (37\%). Concurrently, the fraction of abnormal meiotic forms increased from $23 \%$ to $54 \%$ between day 3 and day 6 of follicle culture. Thus, oocyte quality, as determined by the ability to complete meiosis, deteriorated after 3 days of follicle culture. The arrest of meiosis at MI suggests that the mechanism governing progression from MI to MII is somehow impaired as a result of changes in follicular metabolism between day 3 and day 6 of culture. This result is interesting in light of the observations of follicles from prepubertal animals showing that steroidogenic and glycolytic activities continue to increase over the same period, indicating that follicular metabolism in vitro mimics that in vivo (Boland et al., 1993, 1994a, b; Boland and Gosden, 1994). While nothing is known about the metabolic state of the oocyte during follicle culture, it is likely that breakdown in somatic cell-germ-cell interactions would compromise the provision or utilization of energy substrates required for the completion of meiotic maturation. Alternatively, the failure to transit $\mathrm{MI}$ in cultured oocytes may be due to the loss of critical regulators of cell cycle progression. In this context, it has been shown that oocytes acquire meiotic competence in two steps, first the capacity to initiate meiosis and later the capacity to complete MI and progress to MII (Sorensen and Wassarman, 1976; Wickramasinghe et al., 1991), and that centrosome phosphorylation is required for the full expression of meiotic competence (Wickramasinghe and Albertini, 1992). Thus, inadequate provision or delivery of ATP could impede the cascade of phosphorylation events associated with meiotic maturation. Clearly, the metabolic state of the oocyte during this period of follicle culture needs to be examined.

Equally important for follicular differentiation is the expression of $\mathrm{LH}$ receptors required in vivo for ovulation induction, the stimulation of meiosis and follicular luteinization. Human LH has been shown to produce ovulation in approximately $30 \%$ of cultured follicles from 32-day-old mice (Boland et al., 1993). However, in the study reported here and those of Hartshorne (1994) and Merriman (1993) who used younger mice (10-24 days old), the ovulation rate was lower than $10 \%$. However, other indicators of $\mathrm{LH}$ responsiveness have been reported. Boland et al. (1994b) show that L.H stimulates glucose metabolism and decreases oestradiol synthesis. However, progesterone production has not been detected in $\mathrm{LH}$-treated follicle cultures (Boland et al., 1993). The results presented here are the first to demonstrate clearly an effect of LH on the resumption of meiosis by fluorescence in follicle-enclosed oocytes. The addition of hLH caused meiotic progression in $16 \%$ and $27 \%$ of oocytes in day 2 and day 4 follicle cultures, respectively. However, only $13 \%$ of oocytes progressed from day 6 follicles exposed to hLH, suggesting that responsiveness to $\mathrm{hLH}$ decreases between day 4 and day 6 of culture. Whether the failure to resume meiosis with prolonged culture in response to $h \mathrm{LH}$ is due to a loss of receptors or a failure of meiosis due to factors intrinsic to oocyte metabolism remains unclear.

Attempts to fertilize oocytes from mouse follicles cultured for up to 5-6 days have shown that approximately $41 \%$ of oocytes can cleave and develop to blastocysts if cumulus complexes are cultured with FSH, epidermal growth factor and fetal calf serum for $24 \mathrm{~h}$ before insemination (Spears et al., 1994). In the study reported here, $40 \%$ of cultured oocytes from follicles cultured for 6 days progressed to MI or MII, suggesting that this represents the viable and competent fraction that could give rise to embryos from in vitro fertilization. The successful propagation of a mouse from in vitro fertilization and embryo transfer of an oocyte from a cultured follicle from a 32-day-old mouse attests to the possibility of generating developmentally competent oocytes from this culture system (Spears et al., 1994). However, oocyte quality may have accounted for the low success rate of in vitro fertilization in follicles cultured for 12 days from 10-day-old mice, in which only $10 \%$ of oocytes that developed a polar body cleaved to the two-cell stage (Merriman et al., 1993). 
Collectively, the results of the study reported here indicate that, while various aspects of follicular function are supported in this culture system, the quality of oocytes may be adversely affected by extended culture. To improve the development of viable oocytes within cultured follicles, and to evaluate the metabolic functional attributes of enclosed oocytes, future studies of both nuclear and cytoplasmic maturation (Eppig et al., 1994) will be needed.

This work has been supported as part of the National Cooperative Program on Non-Human In Vitro Fertilization and Preimplantation Development and was funded by the National Institute of Child Health and Human Development, NIH, through cooperative agreement HD 21988. The research was also supported in part by grant HD 20068 to D. F. Albertini from the National Institute of Child Health and Human Development, and $\mathrm{NIH}$ Public Health Services grant P51RR00168-33 awarded to the New England Regional Primate Research Center. The authors thank J. Eppig and R. Foote for valuable comments on the manuscript, and J. Armstrong for preparation of the illustrations. Animals used in this study were maintained in accordance with the guidelines of the Committee on Animals of the Harvard Medical School and those prepared by the Committee on Care and Use of Laboratory Animal Resources, National Research Council [DHHS publication No. (NIH) 85-23, revised 1985].

\section{References}

Biggers JD and Papaioannou VE (1991) Water-escape time in adult mice derived from manipulated preimplantation embryos Journal of In Vitro Fertilization and Embryo Transfer $8352-360$

Boland NI and Gosden RG (1994) Effects of epidermal growth factor on the growth and differentiation of cultured mouse follicles Journal of Reproduction and Fertility 101 369-374

Boland NI, Humpherson PG, Leese HJ and Gosden RG (1993) Pattern of lactate production and steroidogenesis during growth and maturation of mouse ovarian follicles in vitro Biology of Reproduction 48 798-806

Boland NI, Humpherson PG, Leese HJ and Gosden RG (1994a) Characterization of follicular energy metabolism Human Reproduction 9 604-609

Boland NI, Humpherson PG, Leese HJ and Gosden RG (1994b) The effect of glucose metabolism on murine follicle development and steroidogenesis Human Reproduction 9 617-623

Brown D and Rothery P (1993) Structured stochastic models: models with both deterministic and stochastic elements. In Models in Biology pp 123-158. Wiley, New York

Chambers JM, Cleveland WS, Kleiner B and Tukey PA (Eds) (1983) Portraying the distribution of a set of data. Graphical Methods for Data Analysis pp 9-46. Wadsworth, Belmont, CA
Clogg CC and Shihadeh ES (1994) Logit-type regression models for ordinal dependent variables. In Statistical Models for Ordinal Variables pp 139-176. Sage Publications, Thousand Oaks, CA

Draper NR and Smith H (1981) More complicated models. In Applied Regression Analysis (2nd Edn) pp 218-293. Wiley, New York

Eppig J and Schroeder AC (1989) Capacity of mouse oocytes from preantral follicles to undergo embryogenesis and development to live young after growth, maturation, and fertilization in vitro Biology of Reproduction $\mathbf{4 1}$ 268-279

Eppig JJ, Schultz RM, O'Brien M and Chesnel F (1994) Relationship between the development programs controlling nuclear and cytoplasmic maturation of mouse oocytes Developmental Biology 164 1-9

Fieller EC (1944) A fundamental formula in the statistics of biological assay, and some applications Quarterly Journal of Pharmacology 17 117-123

Hartshorne GM, Sargent IL and Barlow DH (1994) Meiotic progression of mouse oocytes throughout follicle growth and ovulation in vitro Human Reproduction 9 352-359

Hirii KF (1992) Exact distributions for polytomous data Journal of the American Statistical Association 87 487-492

Hosmer DW and Lemeshow S (1989) Assessing the fit of the model. In Applied Logistic Regression pp 135-175. Wiley, New York

McCullagh P and Nelder JA (1989) Models for polytomous data. In Generalized Linear Models (2nd Edn) pp 149-192. Chapman and Hall, London

Mattson BM and Albertini DF (1990) Oogenesis: chromatin and microtubule dynamics during meiotic prophase Molecular Reproduction and Development 25 374-383

Merriman JA, Carroll J and Whittingham DG (1993) Maturation and fertilization of oocytes grown in vitro and in vivo Journal of Reproduction and Fertility Abstract Series 11 Abstract I

Nayudu PL and Osborn SM (1992) Factors influencing the rate of preantral and antral growth of mouse ovarian follicles in vitro Journal of Reproduction and Fertility 95 349-362

Qvist R, Blackwell LF, Bourne H and Brown JB (1990) Development of mouse ovarian follicles from primary to preovulatory stages in vitro Journal of Reproduction and Fertility $89169-180$

Rao CR (1965) The theory of least squares when the parameters are stochastic and its application to the analysis of growth curves Biometrika $\mathbf{5 2}$ $447-458$

Sorenson R and Wassarman P (1976) Relationship between growth and meiotic maturation of mouse oocytes Developmental Biology 50 531-536

Spears N, Boland Nl, Murray AA and Gosden RG (1994) Mouse oocytes derived from in vitro grown primary ovarian follicles are fertile Human Reproduction 9 527-532

Wickramasinghe D and Albertini DF (1992) Centrosome phosphorylation and the development and expression of meiotic competence in mouse oocytes Developmental Biology 152 62-74

Wickramasinghe D, Ebert KM and Albertini DF (1991) Meiotic competence acquisition is associated with the appearance of M-phase characteristics in growing mouse oocytes Developmental Biology $143 \quad 162-172$

Wishart J (1938) Growth-rate determination in nutrition studies with the bacon pig, and their analysis Biometrika 30 16-28 